\title{
Calibration and Uncertainty Analysis of Sample-Time Error on High Jitter of Samplers
}

\author{
Chihyun Cho* $\cdot$ Joo-Gwang Lee $\cdot$ Tae-Weon Kang $\cdot$ No-Weon Kang
}

\begin{abstract}
In this paper, we propose an estimation method using multiple in-phase and quadrature (IQ) signals of different frequencies to evaluate the sample-time errors in the sampling oscilloscope. The estimator is implemented by ODRPACK, and a novel iteration scheme is applied to achieve fast convergence without any prior information. Monte-Carlo simulation is conducted to confirm the proposed method. It clearly shows that the multiple IQ approach achieves more accurate results compared to the conventional method. Finally, the criteria for the frequency selection and the signal capture time are investigated.
\end{abstract}

Key Words: Jitter, Sampling Oscilloscope, Time Base Distortion (TBD), Uncertainty, Waveform.

\section{INTRODUCTION}

Recently, a bandwidth required for measurement of a signal has been greatly increased in wireless communications and signal integrity. For example, USB 3.1 requires the measurement bandwidth of approximately $25 \mathrm{GHz}$. Thus, an oscilloscope capable of measuring a wideband signal is getting much attention. The oscilloscope that can measure these high frequency signals is divided into "real-time" and "sampling." The traceable calibration methods for the real-time oscilloscope can be found in [1-5].

A sampling oscilloscope operates on the basis of the principle of "equivalent time sampling," and can digitize a fast signal of up to $100 \mathrm{GHz}$ with a sub-picosecond sampling rate. However, this apparatus inherently has the systematic error time base distortion (TBD) in the sampling time [6].

There are various methods to calibrate TBD in the sampling oscilloscope such as "zero crossing," "sine-fit," and "analytic signal." Among these, the sine-fit method is widely used due to its simplicity and robustness [7]. In-phase and quadrature (IQ) signals are usually fed into additional samplers (usually referred as the reference channel) to estimate TBD [8-10]. Recently, the sample-time error has been considered as "errors in variable" problem and is estimated using a software package for orthogonal distance regression (ODR), ODRPACK [11]. Thus, this method can calibrate TBD as well as random errors and does not require additional compensation for the random jitter, such as in [12].

Some apparatus show high jitter between samplers [13]. Fig. 1 presents the IQ signal measured on various apparatus, and the measurement is mapped to the IQ domain. Therefore, the time information is eliminated and only the voltage value of each channel remains.

When the noise or the uncorrelated jitter between two channels becomes large, the line thickness of the circle becomes thicker. The method in [11] shows inaccurate results, as the non-common timing error increases, such as that shown in Fig. $1(\mathrm{~b})$, since it assumes that the produced sample-time errors are

Manuscript received February 27, 2018 ; Revised April 5, 2018 ; Accepted April 15, 2018. (ID No. 20180227-025J)

Center for Electromagnetic Metrology, Korea Research Institute of Standard and Science, Daejeon, Korea.

"Corresponding Author: Chihyun Cho (e-mail: chihyun.cho@kriss.re.kr)

This is an Open-Access article distributed under the terms of the Creative Commons Attribution Non-Commercial License (http://creativecommons.org/licenses/by-nc/4.0) which permits unrestricted non-commercial use, distribution, and reproduction in any medium, provided the original work is properly cited.

(c) Copyright The Korean Institute of Electromagnetic Engineering and Science. All Rights Reserved. 


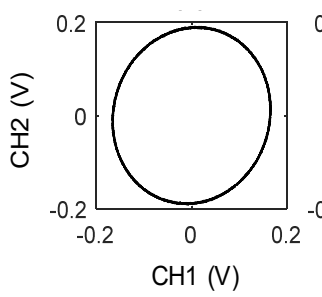

(a)

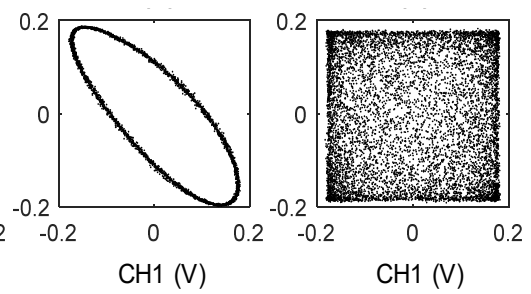

(b)
Fig. 1. Comparison of IQ signals on different sampling oscilloscopes. Each apparatus has (a) low sampler jitter, (b) high sampler jitter, and (c) uncorrelated sampling time between samplers (Note that some sampler modules do not simultaneously measured on the same strobe pulse. In this case, the timing error cannot be estimated using 'sine-fit' method).

almost the same on all reference channels.

In this paper, we propose an estimation method using multiple IQ signals of different frequencies to robustly calibrate TBD as well as random errors with relatively large independent timing errors. The multiple IQ signals have already been used in [8, 11], but they have only been adapted to reduce the ambiguity between TBD and the non-linearity of a sampler or to estimate the initial TBD. However, the proposed method can more accurately correct the nonlinearity of the sampler and the signal source by including the harmonic terms in the fitting model and it does not require the initial values in the fitting process. Unlike conventional methods, the simultaneously measured multiple IQ signals are used to minimize random errors by increasing the orthogonality of the basic functions in the fitting model.

This paper is organized in the following manner: Section II explains the error model for the systematic timing error in the sampling oscilloscope, the multiple IQ approach, and the uncertainty analysis. Section III provides optimum conditions, such as frequency selection on multiple IQ signals. Section IV presents the conclusion.

\section{II . ESTIMATION OF SAMPLE-TIME ERROR}

\section{Error Model}

The produced sample-time errors on each channel are composed of a systematic error $T B D$ and random error $\tau_{\text {sampler }}$ (jitter of sampler), $\tau_{\text {source }}$ (jitter of source), and $\tau_{\text {trigger }}$ (jitter of triggering circuitry), as shown in Fig. 2.

$$
\Delta t_{j, i}=T B D_{i}+\tau_{(\text {sampler }) j, i}+\tau_{(\text {source }) j, i}+\tau_{(\text {trigger }) i},
$$

where $j$ and $i$ indicate the $j^{\text {th }}$ channel and $i^{\text {th }}$ sample index, respectively. If $\tau_{\text {(sampler);i } i}$ and $\tau_{(\text {source) } ; i}$ are smaller than others, the timing errors $\Delta t_{j, i}$ can be approximated to $T B D_{i}+\tau_{\text {(trigger }) \text { i. }}$ Thus, all channels have a common timing error that can be estimated using the IQ signal.

However, if the non-common error $\tau_{(\text {sampler }) ; i}$ and $\tau_{(\text {source }) ; i}$ are

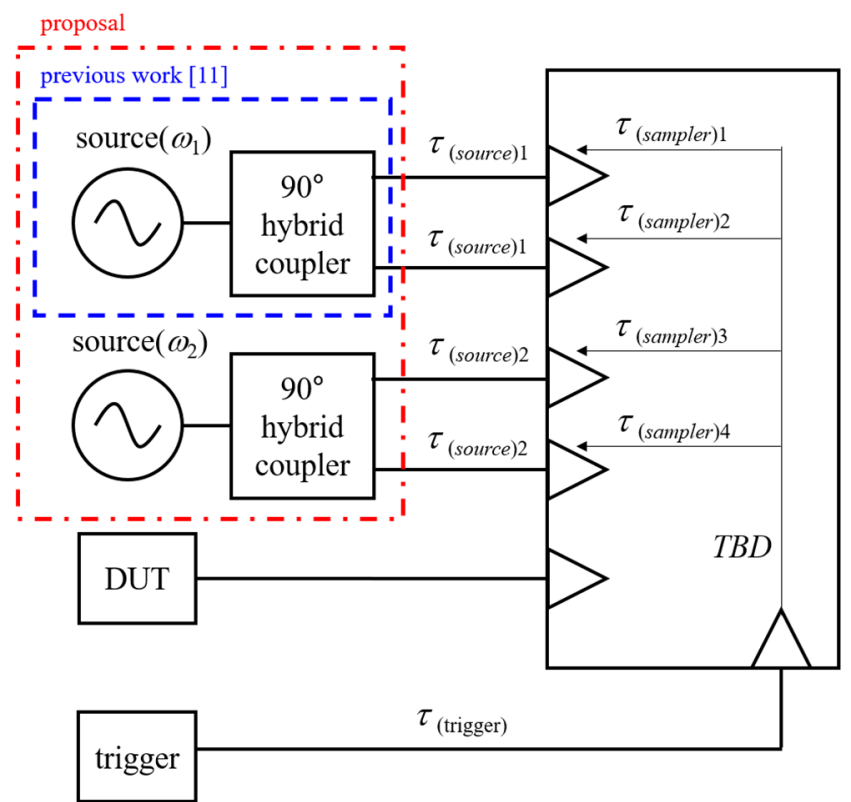

Fig. 2. Model of the sample-time error for the sampling oscilloscope.

relatively larger than other errors, it cannot be estimated with the single IQ signal. In this case, multiple IQ signals are required to accurately estimate the common errors in the measurements. The regression is realized by the ODR, as explained in the next section. To suppress ambiguity of the measurement, the selected frequency is a slightly different frequency (this will be discussed in detail in Section III).

\section{Estimation of Sample Time}

The following fitting function is used in the estimation of the sample-time error:

$$
y_{j, i}=\sum_{h=1}^{N}\left(a_{h, j} \cos \left(h \omega_{j}\left(t_{i}+\delta_{i}\right)\right)-b_{h, j} \sin \left(h \omega_{j}\left(t_{i}+\delta_{i}\right)\right)\right)+c_{j}+\varepsilon_{j, i},
$$

where $\delta_{i}$ represents the summation of common timing errors $T B D_{i}$ and $\tau_{\text {(trigger })_{i} .} h$ means the number of harmonics generated by the source and non-linearity of the sampler, $c_{j}$ is the offset of the sampler, and $\varepsilon_{j, i}$ represents the residual errors with zero mean.

We estimate the timing error with the iterated ODR. The iterated ODR makes that $\omega_{\delta} \sigma_{\delta}^{2}$ and $\omega_{\varepsilon} \sigma_{\varepsilon}^{2}$ have the smallest values in the regression, where $\omega_{\varepsilon}$ and $\omega_{\delta}$ is the weighting vector. The initial values $a_{b, j}, b_{b, j}$, and $c_{j}$ are found by the linear least square fitting with $\delta_{i}=0$. Then, the weighting vector $\omega_{\varepsilon}$ is adjusted so that $\omega_{\delta} \sigma_{\delta}^{2} / \omega_{\varepsilon} \sigma_{\varepsilon}^{2}$ is between 0.99 and 1.01 , while $\omega_{\delta}$ is set to 1 . In each adjustment, the values found in the previous step are used as the initial values in the current step. The new weighting vector $\omega_{\varepsilon}$ is defined in the following manner:

$$
\omega_{\varepsilon, k}=\omega_{\varepsilon, k-1}\left(\sigma_{\delta, k-1}^{2} / \sigma_{\omega, k-1}^{2}\right)^{G} .
$$


Accordingly, $G$ has the value of either +1 or -1 , as $\omega_{\delta} \sigma_{\delta}^{2} / \omega_{\varepsilon} \sigma_{\varepsilon}^{2}$ is far away from 1 . This approach can enable fast convergence and achieve high accuracy. In addition, it does not require initial TBD, which are usually estimated on slightly different frequencies to increase accuracy [9]. The performance of the proposed method is compared with the Time Base Correction (TBC) software [14], which implements the algorithm in [11]. In the Monte-Carlo simulation, $3 \mathrm{GHz}$ and $3.3 \mathrm{GHz}$ are used as multiple quadrature signals that have the amplitude of $0.4 \mathrm{~V}$. Note that optimum conditions on frequency selection will be considered in Section III. The second and third harmonics are $1.5 \%$ and $0.25 \%$ of fundamental signals. The total epoch is $10 \mathrm{~ns}$, with the sampling interval of $5 \mathrm{ps}$. The random errors are set as $\sigma_{T B D}=6.5 \mathrm{ps}$ and $\sigma_{\tau_{\text {source }}}=0.2 \mathrm{ps}$, respectively. Further, 100 data sets are created independently per different sampler jitter and random noise, and the sample-time errors. The estimation results of the proposed method (blue dot) and the TBC software (red asterisk) are depicted in Fig. 3. The y-axis

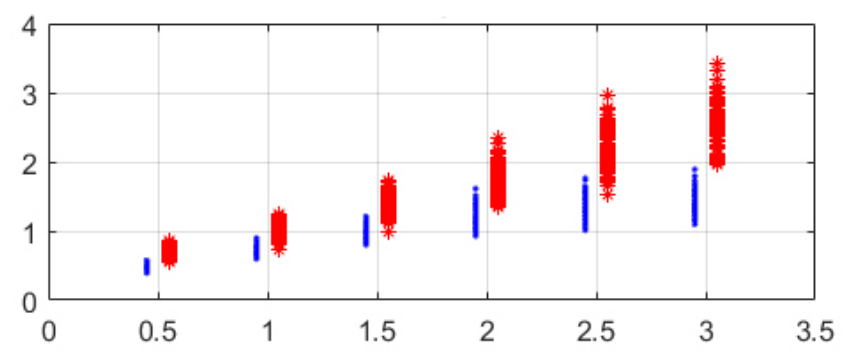

(a)

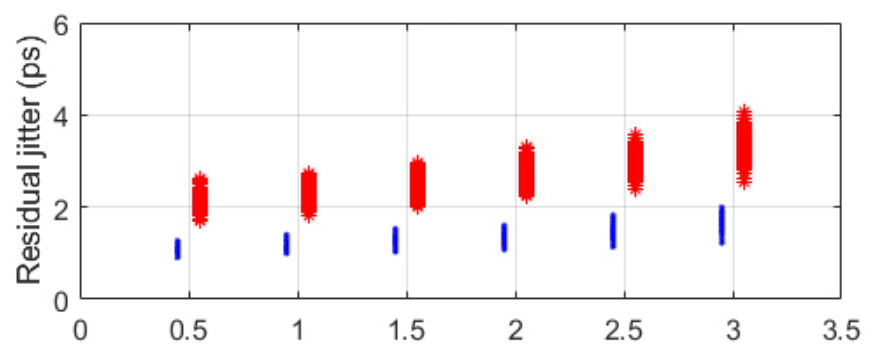

(b)

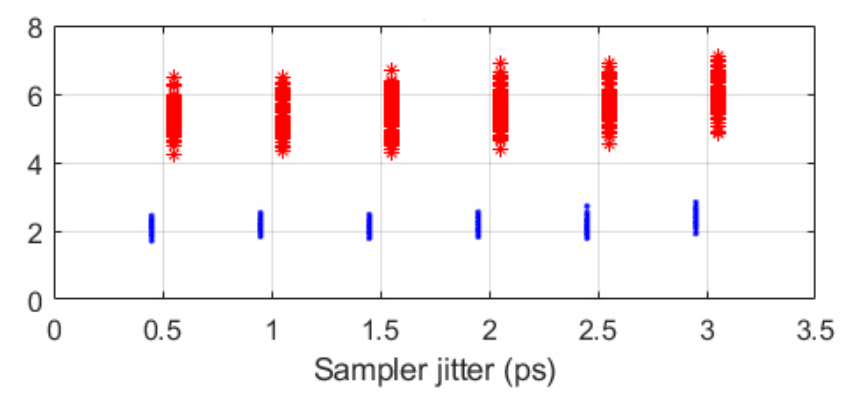

(c)

Fig. 3. Comparison of residual timing error for multiple IQ signals and the TBC [14]. The results of the iterated ODR and the TBC are marked with “." and “*”, respectively. Random noise $\sigma_{\varepsilon}$ are (a) $1 \%$, (b) $4 \%$, and (c) $10 \%$ of $0.4 \mathrm{~V}$ (amplitude of the fundamental signals), respectively. represents the RMS error between the actual and estimated sample-time error. As the sampler jitter increases, the error of estimators also increases due to the increment of non-common errors. Moreover, the result shows that the RMS errors increase when the amplitude noise $\varepsilon$ increases. The proposed method shows more accurate results compared with the TBC software, even if the TBC software uses additional IQ signals measured at different frequencies. This implies that multiple IQ signals measured simultaneously are more useful than the separately measured IQ signals for eliminating the effects of random jitter between samplers.

\section{Uncertainty Analysis}

The uncertainty of the sample time errors in estimators can be considered as the non-linear ordinary least square problems in the following manner [15]:

$$
\begin{gathered}
\mathbf{S}_{\text {param }}=\left(\mathbf{J}^{T} \mathbf{J}\right)^{-1} \sigma^{2} \\
\mathbf{J}=\sqrt{\omega_{\varepsilon}}\left(\begin{array}{ccccc}
\mathbf{A}_{1} & 0 & 0 & 0 & \mathbf{H}_{1} \\
0 & \mathbf{A}_{2} & 0 & 0 & \mathbf{H}_{2} \\
0 & 0 & \mathbf{A}_{3} & 0 & \mathbf{H}_{3} \\
0 & 0 & 0 & \mathbf{A}_{4} & \mathbf{H}_{4} \\
0 & 0 & 0 & 0 & \mathbf{I}_{n} / \sqrt{\omega_{\varepsilon}}
\end{array}\right) .
\end{gathered}
$$

$\mathbf{S}_{\text {param }}$ in (5) is the covariance of the estimated parameters $\left(a_{h, j}\right.$, $\left.b_{b_{j}, j}, c_{j}, \delta_{i}\right)$, and $\sigma^{2}$ is the residual variance of estimators; thus $\sigma^{2}$ $=\left(\omega_{\delta} \sigma_{\delta}^{2}+\omega_{\varepsilon} \sigma_{\varepsilon}^{2}\right) / v$. The degree of freedom $v$ is from $k L-p$, since the observation points are the number of $k L+L$ and the number of estimated parameters is $p+L\left(k L=n\left(\varepsilon_{i j}\right), L=n\left(\delta_{i}\right)\right.$, and $p=n\left(\left\{a_{b, j}, b_{b, j}, c_{j}\right\}\right)$, where $n(\cdot)$ is the number of elements in the set). Note that the degree of freedom is different from [15] and is almost linearly increased as the number of channels $k$ increase. This means that adding a reference channel improves accuracy as well as reduces the confidence interval on the estimating parameters. $\mathbf{I}_{n}$ is an $L \times L$ identity matrix, and $\mathbf{A}_{j}$ and $\mathbf{H}_{j}$ is the Jacobian matrix for each channel with the size $L \times p$ and $L \times L$, respectively:

$$
\begin{gathered}
\mathbf{A}_{j}(n, m)=\frac{\partial\left(Y_{j, n}^{\text {Meas. }}-Y_{j, n}^{\text {fitting }}\right)}{\partial \mathbf{P}_{j}(m)} . \\
\mathbf{H}_{j}(n, n)=\frac{\partial\left(Y_{j, n}^{\text {Meas. }}-Y_{j, n}^{\text {fiting }}\right)}{\partial \delta_{n}} . \\
\mathbf{P}_{j}^{T}=\left(a_{1, j}, \ldots, a_{h, j}, b_{1, j}, \ldots, b_{h, j}, c_{j}\right)^{T} .
\end{gathered}
$$

Thus, $\mathbf{S}_{\text {param }}$ in (5) has the same structure as (10).

$$
\mathbf{S}_{\text {param }}=\left[\begin{array}{cc}
\sigma_{P}^{2} & \sigma_{P} \sigma_{\delta} \\
\sigma_{P} \sigma_{\delta} & \sigma_{\delta}^{2}
\end{array}\right] .
$$

In this matrix, the covariance for the sample-time errors $\mathbf{S}_{t}$ is $\left[\sigma_{\delta}^{2}\right]$. 


\section{OPTIMUM CONDITIONS FOR ESTIMATIONS}

The timing error $\delta$ has a different effect on the measured value $y$ depending on the signal $g$ on $t_{\mathrm{i}}$, as shown below [9]:

$$
\operatorname{var}\left(y_{i}\right) \approx \sigma_{\delta}^{2}\left(g^{\prime}\left(t_{i}\right)^{2}\right)+\sigma_{\varepsilon}^{2},
$$

where $\varepsilon$ is the noise, $\sigma$ is the variance of each variable, and $g^{\prime}\left(t_{i}\right)$ is the derivative of input signal at time $t_{i}$, respectively. Thus, the IQ signal with sine waveforms can produce orthogonal basis functions $\cos \left(\omega_{1} t\right)$ and $\sin \left(\omega_{1} t\right)$ in ODR fitting. The orthogonality of the basis function can be increased more by using the IQ signal with different frequencies. In this section, Monte-Carlo simulations are performed to obtain these optimal conditions. First, the condition for the frequency selection is addressed. Fig. 4 presents the residual estimated timing errors as the frequency of the additional reference channel changes. Here, 10 measurement sets are created, and each set comprises 20,000 data samples. The sample interval is $5 \mathrm{ps}$ and the amplitude of reference signals are the same as those in the previous section. The $3 \mathrm{GHz}$ and $11 \mathrm{GHz}$ signals are used as the first reference signals in Fig. 4(a) and (b), respectively. In each Monte-Carlo simulation, the random errors are differently changed to achieve independence; $\sigma_{T B D}=6.5 \mathrm{ps}, \sigma_{\tau_{\text {sampler }}}=2.5 \mathrm{ps}, \sigma_{\tau_{\text {source }}}=0.2 \mathrm{ps}, \sigma_{\varepsilon}=0.004 \mathrm{~V}$ in Fig. $4(\mathrm{a})$ and $\sigma_{T B D}=3.0 \mathrm{ps}, \sigma_{\tau_{\text {sampler }}}=1.0 \mathrm{ps}, \sigma_{\tau_{\text {source }}}=0.2$ ps, $\sigma_{\varepsilon}=0.008 \mathrm{~V}$ in Fig. 4(b). In the simulation, the result using the same frequency as the first reference signal also shows small

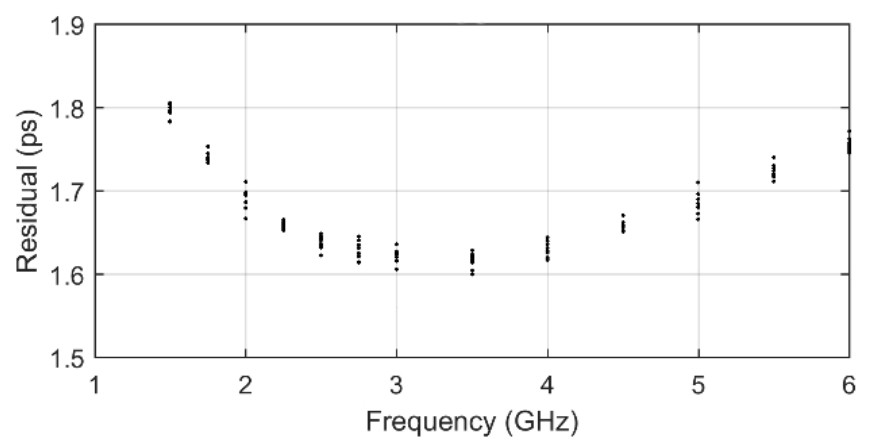

(a)

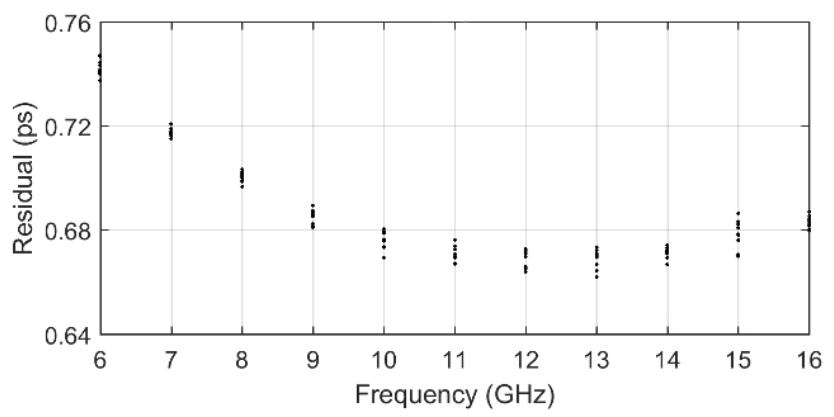

(b)

Fig. 4. The residual timing errors as a function of frequency of second reference signal. First reference signal is (a) $3 \mathrm{GHz}$, and (b) $11 \mathrm{GHz}$.

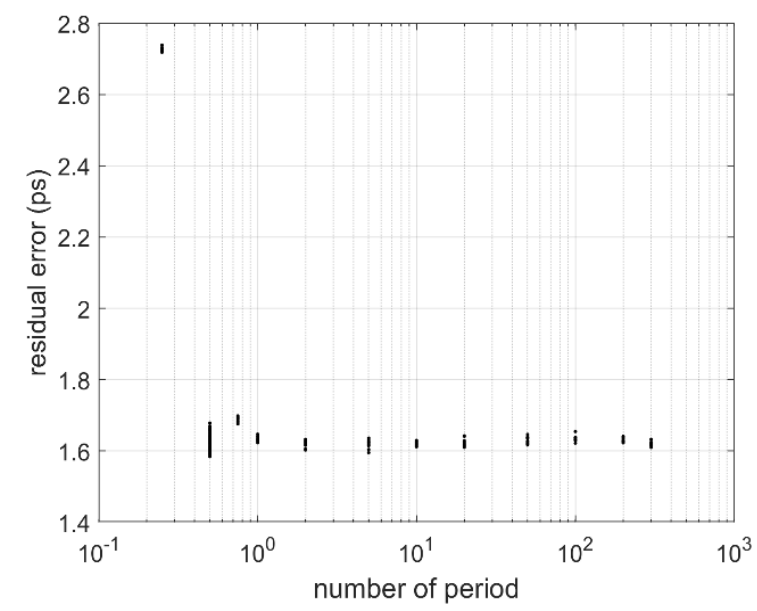

Fig. 5. The residual timing errors as a function of number of periods on the measurement of reference signal.

residual errors that are different from the result in [8], since the proposed approach yields sample-time errors, including random errors, in addition to TBD. In both results, RMS residual errors are the smallest when the frequency of the second signal is approximately 1.1 times greater than the first reference signal.

Next, the criterion for the number of periods is investigated. All conditions are the same as the simulation in Fig. 4(a), and the sample time is determined as $N_{\text {period }} / L / f_{\text {reference. }} N_{\text {period }}$ is the number of periods and $f_{\text {reference }}$ is the frequency of the first reference signal. In this simulation, the frequency of the first and second frequencies is set as 3 and $3.5 \mathrm{GHz}$, and the result is represented in Fig. 5. When the period is less than 0.5, the sine waveforms measured by reference channels are too short to be orthogonal to each other. However, the estimator can obtain a good estimation result when the reference signal is captured for at least one period on the oscilloscope display.

\section{CONCLUSION}

In this paper, we propose an estimation method based on using the multiple IQ signals to evaluate the sample-time errors in the sampling oscilloscope. The estimator is implemented using the ODR and does not require any prior information. The numerical simulations reveal that the proposed method is more accurate than the method using only a single IQ signal when the independent jitter between the samplers is increased. It is also found that the frequency of the second reference and quadrature signals should be selected to be 1.1 times higher than the frequency of first ones. Moreover, the signals of approximately one period have to be captured on an oscilloscope display to adequately calibrate sample-time errors.

This research was supported by the Physical Metrology for National Strategic Needs funded by Korea Research Institute of Standards and Science (No. KRISS-2018-GP2018-0005). 


\section{REFERENCES}

[1] D. A. Humphreys, M. Hudlicka, and I. Fatadin, "Calibration of wideband digital real-time oscilloscopes," IEEE Transactions on Instrumentation and Measurement, vol. 64, no. 6, pp. 1716-1725, 2015.

[2] C. Cho, J. G. Lee, J. H. Kim, and D. C. Kim, "Uncertainty analysis in EVM measurement using a Monte Carlo simulation," IEEE Transactions on Instrumentation and Measurement, vol. 64, no. 6, pp. 1413-1418, 2015.

[3] C. Cho, J. G. Lee, P. D. Hale, J. A. Jargon, P. Jeavons, J. Schlager, and A. Dienstfrey, "Calibration of channel mismatch in time-interleaved real-time digital oscilloscopes," in Proceedings of 2015 85th Microwave Measurement Conference (ARFTG), Phoenix, AZ, 2015, pp. 1-5.

[4] C. Cho, J. G. Lee, P. D. Hale, J. A. Jargon, P. Jeavons, J. B. Schlager, and A. Dienstfrey, "Calibration of time-interleaved errors in digital real-time oscilloscopes," IEEE Transactions on Microwave Theory and Techniques, vol. 64, no. 11, pp. 4071-4079, 2016.

[5] D. Kim, J. G. Lee, D. J. Lee, and C. Cho, "Traceable calibration for a digital real-time oscilloscope with time interleaving architecture," Measurement Science and Technology, vol.29, no. 1, article ID. 015003, 2017.

[6] J. B. Rettig and L. Dobos, "Picosecond time interval measurements," IEEE Transactions on Instrumentation and Measurement, vol. 44, no. 2, pp. 284-287, 1995.

[7] IEEE Standard for Digitizing Waveform Recorders, IEEE Standard 1057-2007 (Revision of IEEE 1057-1994), 2008.

[8] G. N. Stenbakken and J. P. Deyst, "Time-base nonlinearity determination using iterated sine-fit analysis," IEEE Transactions on Instrumentation and Measurement, vol. 47, no. 5, pp.

\section{Chihyun Cho}

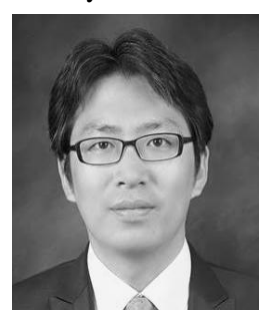

received the B.S., M.S., and Ph.D. degrees in electronic and electrical engineering from Hongik University, Seoul, Korea, in 2004, 2006, and 2009, respectively. From 2009 to 2012, he participated in the development of military communication systems at the Communication R\&D Center, Samsung Thales. Since 2012, he has been with the Korea Research Institute of Standards and Science (KRISS) in Daejeon. In 2014, he was a guest researcher at the National Institute of Standards and Technology (NIST) in Boulder, CO, USA. He also served on the Presidential Advisory Council on Science and Technology (PACST) in Korea from 2016 to 2017. His current research interests include microwave metrology, time domain measurement, and pulse and digital parameter standards.
1056-1061, 1998.

[9] C. M. Wang, P. D. Hale, and K. J. Coakley, "Least-squares estimation of time-base distortion of sampling oscilloscopes," IEEE Transactions on Instrumentation and Measurement, vol. 48, no. 6, pp. 1324-1332, 1999.

[10] D. A. Humphreys, "Vector measurement of modulated RF signals by an in-phase and quadrature referencing technique," IEE Proceedings-Science, Measurement and Technology, vol. 153, no. 6, pp. 210-215, 2006.

[11] P. D. Hale, C. M. Wang, D. F. Williams, K. A. Remley, and J. D. Wepman, "Compensation of random and systematic timing errors in sampling oscilloscopes," IEEE Transactions on Instrumentation and Measurement, vol. 55, no. 6, pp. 2146-2154, 2006.

[12] P. D. Hale, T. S. Clement, K. J. Coakley, C. M. Wang, D. C. DeGroot, and A. P. Verdoni, "Estimating the magnitude and phase response of a $50 \mathrm{GHz}$ sampling oscilloscope using the 'nose-to-nose' method," in Proceedings of the 55th ARFTG Conference Digest, Boston, MA, 2000, pp. 1-8.

[13] D. A. Humphreys and M. Akmal, "Channel timebase errors for digital sampling oscilloscopes," in Proceedings of 2012 Conference on Precision Electromagnetic Measurements (CPEM), Washington, DC, 2012, pp. 520-521.

[14] National Institute of Standards and Technology, "Timebase correction software," [Online]. Available: https:// www.nist.gov/services-resources/software/timebase-correction-software.

[15] C. M. Wang, P. D. Hale, and D. F. Williams, "Uncertainty of timebase corrections," IEEE Transactions on Instrumentation and Measurement, vol. 58, no. 10, pp. 3468-3472, 2009.

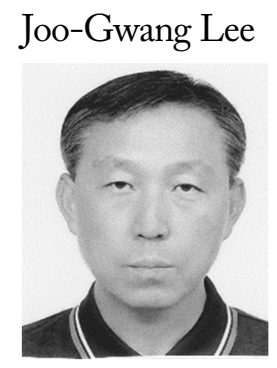

received the B.S. degree in electronic engineering from Hanyang University, Seoul, Korea in 1984 and the M.S. and $\mathrm{Ph} . \mathrm{D}$. degrees from the Korea Advanced Institute of Science and Technology (KAIST), Daejeon, Korea in 1994 and 2000, respectively. Since 1986, he has been with the Korea Research Institute of Standards and Science (KRISS), Daejeon, Korea. His research interests include radiofrequency and microwave measurements, time-domain metrology, and electromagnetic compatibility. 


\section{Tae-Weon Kang}

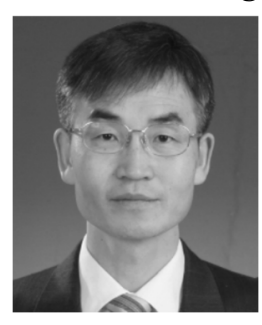

received the B.S. degree in electronics engineering from Kyungpook National University, Daegu, Korea, in 1988, and the M.S. and the Ph.D. degrees in electronic and electrical engineering from the Pohang University of Science and Technology, Pohang, Korea, in 1990 and 2001, respectively. He joined the Center for Electromagnetic Wave, Korea Research Institute of Standards and Science (KRISS), Daejeon, Korea, in 1990. In 2002, he spent a year as a Visiting Researcher under the Korea Science and Engineering Foundation for Post-Doctoral Fellowship Program with the George Green Institute for Electromagnetics Research, University of Nottingham, Nottingham, UK, where he was involved in measurement of absorbing performance of electromagnetic absorbers and a generalized transmission line modeling method. He has been involved in electromagnetic metrology and is currently a Principal Research Scientist. His current research interests include electromagnetic metrology, such as electromagnetic power, noise, and antenna characteristics, and numerical modeling in electromagnetic compatibility.

\section{No-Weon Kang}

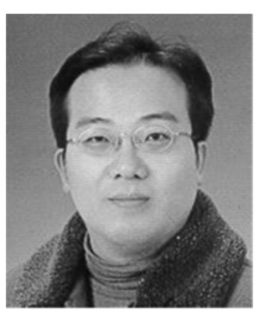

received the B.S., M.S., and Ph.D. degrees from Seoul National University, Seoul, Korea, in 1991, 1994, and 2004, respectively, all in electrical engineering. His $\mathrm{Ph} . \mathrm{D}$. degree was focused on numerical analysis centered in the finite-difference timedomain (FDTD) method, in particular, an algorithm that reconstructs the complex permittivity profile of unknown scatterers. He was with the Electromagnetic Apparatus Laboratory, LG Industrial Systems, Seoul, from 1994 to 1999, where he was involved in static magnetic field analysis of electrical apparatus. He has been with the Korea Research Institute of Standards and Science (KRISS), Daejeon, Korea, since 2004, where he is currently the Head of the Center for electromagnetic metrology and a Principal Research Scientist in electromagnetic metrology. His current research interests include electromagnetic field strength, antenna measurement standards, electromagnetic interference/electromagnetic interference issues, and electro-optic sensor. 